\title{
microRNA-126 suppresses PAK4 expression in ovarian cancer SKOV3 cells
}

\author{
PING LUO ${ }^{1}$, JING FEI $^{2}$, JIANWEI ZHOU ${ }^{2}$ and WEIJIANG ZHANG ${ }^{2}$ \\ ${ }^{1}$ Department of Gynecology, Fuyang People's Hospital, Hangzhou, Zhejiang 311400; ${ }^{2}$ Department of Gynecology, \\ The Second Affiliated Hospital, School of Medicine, Zhejiang University, Hangzhou, Zhejiang 310009, P.R. China
}

Received September 7, 2014; Accepted January 29, 2015

DOI: $10.3892 / \mathrm{ol} .2015 .3012$

\begin{abstract}
Primary ovarian cancer is one of the predominant causes of mortality from gynecological cancer. The suppression of serine/threonine p21-activated kinases (PAKs), proteins involved in cell morphology and cytoskeletal reorganization, has been hypothesized to improve the survival of patients with ovarian cancer. However, the association between microRNA-126 (miR-126) and PAK4 in the inhibition of ovarian cancer cell invasion remains to be established. The present study demonstrated changes in the level of PAK4 expression in ovarian cancer SKOV3 cells with altered miR-126 compared with normal SKOV3 cells. The SKOV3 cells that were transfected with LV3-miR-126 to increase miR-126 expression exhibited significantly downregulated expression levels of PAK4 $(\mathrm{P}<0.05)$, whilst transfection with the LV3-hsa-miR-126 inhibitor increased the expression of PAK4 in these cells $(\mathrm{P}<0.05)$, as assessed by immunofluorescence staining. Furthermore, western blot analysis revealed a significant increase in PAK4 expression in the SKOV3 cells transfected with the LV3-hsa-miR-126 inhibitor, and a decrease in those transfected with LV3-hsa-miR-126. The present study provides an experimental foundation for miR-126 as a potential tumor suppressor that may decrease PAK4 expression to inhibit ovarian cancer cells.
\end{abstract}

\section{Introduction}

Epithelial ovarian cancer is one of the most common causes of mortality among females (1). The high mortality rate of ovarian cancer patients (9.30 out of every 100,000 patients each year) is a consequence of late-stage diagnosis, and the five-year survival rate $(<50 \%$ for patients $>64$ years) for the advanced stages is extremely poor in the USA, Europe and

Correspondence to: Dr Weijiang Zhang, Department of Gynecology, The Second Affiliated Hospital, School of Medicine, Zhejiang University, 88 Jiefang Road, Hangzhou, Zhejiang 310009, P.R. China

E-mail: zhou_jianwei@126.com

Key words: microRNA-126, p21-activated kinase 4, ovarian cancer
Japan (2). A large tumor burden and extensive metastatic lesions of the abdominal cavity also contribute to the poor prognosis and the high rate of mortality of this disease (3). Tumor cell migration/invasion is a complex process involving cytoskeletal reorganization and membrane ruffling. The suppression of cytoskeletal reorganization and the redistribution of actin fibers may lead to the formation of non-adhesive membrane protrusions and therefore, dysregulated cellular adhesion capacity; this has been hypothesized to improve the survival of patients with ovarian cancer (4).

The actin cytoskeleton is essential for cell motility and cell invasion $(5,6)$. Serine/threonine p21-activated kinases (PAKs) are effector proteins for the Rho GTPases Cdc42 and Rac, which are important for cell morphology and cytoskeletal reorganization $(7,8)$. PAK4 was initially identified due to its regulation of cytoskeletal reorganization $(9,10)$. Subsequent studies indicated that PAK4 is a key integrator of cell migration, invasion and apoptosis $(11,12)$. Furthermore, PAK4 is upregulated in the majority of cancer cell lines, while previous studies have revealed that PAK4 is strongly linked to the progression of ovarian tumors and breast cancer. Additionally, overexpression of PAK4 in mammary epithelial cells leads to tumorigenesis in mice. Therefore, this protein may be a valuable molecular prognostic marker and therapeutic target in a number of cancers (13-16).

microRNAs (miRNA/miR), are non-coding RNAs of $\sim 22$ nucleotides, and are involved in various cellular processes, including proliferation, differentiation, apoptosis and invasion (17-19). miR-126 originates from a common precursor structure located within the EGFL7 gene, and its expression levels have been reported to vary in a number of human cancers; patients with low miR-126 expression exhibit poor survival compared with patients with high miR-126 levels (20-23). It has been proposed that miR-126 is essential in the inhibition of the invasive growth of cancer cells. Thus, the current study investigated whether the up- or downregulation of miR-126 modulates PAK4 expression in human ovarian cancer cells.

\section{Materials and methods}

Cell culture. SKOV3 cells (American Type Culture Collection, Rockville, MD, USA) were used as the ovarian cancer cells in the present study. The cells were maintained and propagated in vitro by serial passage in Dulbecco's modified 
Eagle's medium (DMEM; Gibco, Life Technologies Corporation, Carlsbad, CA, USA) supplemented with $10 \%$ fetal bovine serum (FBS; Gibco, Life Technologies Corporation), $100 \mathrm{IU} / \mathrm{ml}$ penicillin and $100 \mu \mathrm{g} / \mathrm{ml}$ streptomycin in a humidified atmosphere of $5 \% \mathrm{CO}_{2}$ at $37^{\circ} \mathrm{C}$. All procedures were performed according to the internationally accepted ethical guidelines and approved by the Institutional Review Board of the Second Affiliated Hospital, School of Medicine, Zhejiang University (Hangzhou, China).

Plasmid construction, lentivirus packaging and cell infection. pGLV3/H1/green fluorescent protein (GFP)+Puro (pGLV3; Shanghai GenePhama Co., Ltd., Shanghai, China), a lentiviral vector, was used to construct the pGLV3-miR-126 plasmid. The miR-126 mimic, miR-126 inhibitor and negative control (NC) oligonucleotides were chemosynthesized by Shanghai GenePhama Co., Ltd. The oligonucleotide sequences were as follows: miR-126, 5'-TCG TACCGTGAGTAATAATGCG-3'; hsa-miR-126 inhibitor, 5'-CGCATTATTACTCACGGTACGA-3'; and microRNA NC, 5'-TTCTCCGAACGTGTCACGT-3'. The miR-126 small hairpin (sh)DNA double chain template sequence was synthesized artificially, and inserted into the pGLV3-miRNA lentivirus plasmid. The miR-126 mimic sequence was constructed as follows: (Forward) hsa-miR-126-BamHI, GATCCGTCGTACCGTGAGTAATAATGCGTTCAAGAG ACGCATTATTACTCACGGTACGACTTTTTTG; (reverse) hsa-miR-126-EcoRI, AATTCAAAAAAGTCGTACCGT GAGTAATAATGCGTCTCTTGAACGCATTATTACTCA CGGTACGACG. The miRNA-126 inhibitor sequence was constructed as follows: (Forward) hsa-miR-126-BamHI, GATCCGAGCATGGCACTCATTATTACGCTTCAAGAG AGCGTAATAATGAGTGCCATGCTCTTTTTTG; (reverse) hsa-miR-126-EcoRI, AATTCAAAAAAGAGCATGGCA CTCATTATTACGCTCTCTTGAAGCGTAATAATGAGTGCC ATGCTCG. pGLV3-shDNA-NC was used as a negative control, with the following sequence: (Forward) NC-BamHI, GATCCGTCGTACCGTGAGTAATAATGCGTTCAAGAG ACGCATTATTACTCACGGTACGACTTTTTTG; (reverse) shNC-EcoRI, AATTCAAAAAAGTCGTACCGTGAGTAA TAATGCGTCTCTTGAACGCATTATTACTCACGGTACG ACG.

The 293T producercell line (Cell Bank of Chinese Academy of Science, Beijing, China) was maintained in DMEM, with $10 \% \mathrm{FBS}, 4.0 \mathrm{mM}$ L-glutamine, $100 \mathrm{U} / \mathrm{ml}$ penicillin and $100 \mu \mathrm{g} / \mathrm{ml}$ streptomycin. One day prior to transfection, the cells were seeded into a $15-\mathrm{cm}$ dish. pGLV3-miR-126 or pGLV3 vectors and packing plasmids, including pGag/Pol, pRev and pVSV-G (Shanghai GenePhama Co., Ltd.) were co-transfected using RNAi-mate (Shanghai GenePhama Co., Ltd.), according to the manufacturer's instruction. At $72 \mathrm{~h}$ post-transfection, the supernatant was harvested, cleared by centrifugation $\left(2,200 \mathrm{x} \mathrm{g}\right.$ at $4^{\circ} \mathrm{C}$ for $\left.4 \mathrm{~min}\right)$, passed through a $0.45-\mu \mathrm{m}$ syringe filter, and cleared by centrifugation again $\left(20,000 \mathrm{rpm}\right.$ at $4^{\circ} \mathrm{C}$ for $\left.2 \mathrm{~h}\right)$. The titer of the virus was measured according to the expression level of GFP, following the manufacturer's instructions. The packaged lentiviruses were designated LV3-hsa-miR-126, LV3-hsa-miR-126 inhibitor and LV3-NC. The sequences of the resulting vectors were verified by sequence analysis.
The SKOV3 cells were infected with LV3-hsa-miR-126, LV3-has-miR-126 inhibitor or LV3-NC, at a multiplicity of infection ratio of 15 , in the presence of $5 \mu \mathrm{g} / \mathrm{ml}$ polybrene (Shanghai GenePhama Co., Ltd.); the infection efficiency was $80-90 \%$, as assessed by microscopic analysis of GFP fluorescence.

Immunofluorescence staining and western blot analysis. At $48 \mathrm{~h}$ post-transfection, the cells were fixed in $4 \%$ paraformaldehyde, washed three times with phosphate-buffered saline (PBS), and incubated for $5 \mathrm{~min}$ at $-20^{\circ} \mathrm{C}$ in $95 \%$ ethanol (vol/vol in PBS). The cells were subsequently washed three times with PBS, blocked for $1 \mathrm{~h}$ in 5\% normal goat serum in PBS with 0.1X Triton X-100, and incubated overnight with polyclonal rabbit anti-human PAK4 antibodies (Abcam, Cambridge, MA, USA; dilution, 1:200) at $4^{\circ} \mathrm{C}$. The following day, the cells were washed three times with PBS and incubated for $40 \mathrm{~min}$ at $37^{\circ} \mathrm{C}$ with the corresponding secondary antibody [polyclonal goat anti-rabbit immunoglobulin (Ig)G (H+L)-tetramethylrhodamine (TRITC); dilution 1:200; SouthernBiotech, Birmingham, AL, USA], then washed and mounted. Immunostained SKOV3 cultures were examined under a laser scanning confocal microscope (LSM 510 Meta; Carl Zeiss Microscopy GmbH, Jena, Germany) for detection of the TRITC-fluorophore. Each group was photographed at x400 magnification with the aid of a digital camera attached to the microscope, and the expression of PAK4 was assessed by calculating the percentage of positive cells and the optical density, subsequent to defining a threshold for background correction.

For the western blot analysis, proteins were extracted from the SKOV3 cells, solubilized in radioimmunoprecipitation assay buffer, separated on $10 \%$ sodium dodecyl sulfate polyacrylamide gel electrophoresis (Wuhan Boster Ltd., Wuhan, China) and electro-transferred onto polyvinylidene difluoride membranes (Invitrogen Life Technologies, Carlsbad, CA, USA). The membranes were blocked in 5\% skimmed milk powder prepared in Tris-buffered saline with Triton X-100 (TBS-T) for $30 \mathrm{~min}$. For PAK4 detection, the membranes were incubated at $4^{\circ} \mathrm{C}$ overnight with anti-PAK4 antibodies (Abcam; dilution 1:500). The membranes were washed three times for 10 min in TBS-T and incubated with a 1:5,000 dilution of horseradish peroxidase-conjugated goat anti-rabbit IgG for $2 \mathrm{~h}$. Finally, the membranes were washed six times for $20 \mathrm{~min}$ each in TBS-T, prior to development with a standard enhanced chemiluminescence kit (KeyGEN Biotech, Nanjing, China). The densitometric analysis of the PAK4 and $\beta$-actin bands was assayed by Quantity One software (Bio-Rad Laboratories, Inc., Hercules, CA, USA).

Statistical analysis. All data are presented as the mean \pm standard deviation (SD). Statistical analysis was performed using SPSS statistical software, version 17.0 (SPSS, Inc., Chicago, IL, USA) for Windows. The significance of any differences between groups was evaluated using one-way analysis of variance. $\mathrm{P}<0.05$ was considered to indicate a statistically significant difference.

\section{Results}

Immunofluorescence double staining and semi-quantitative confocal laser scanning analysis detected the expression of 

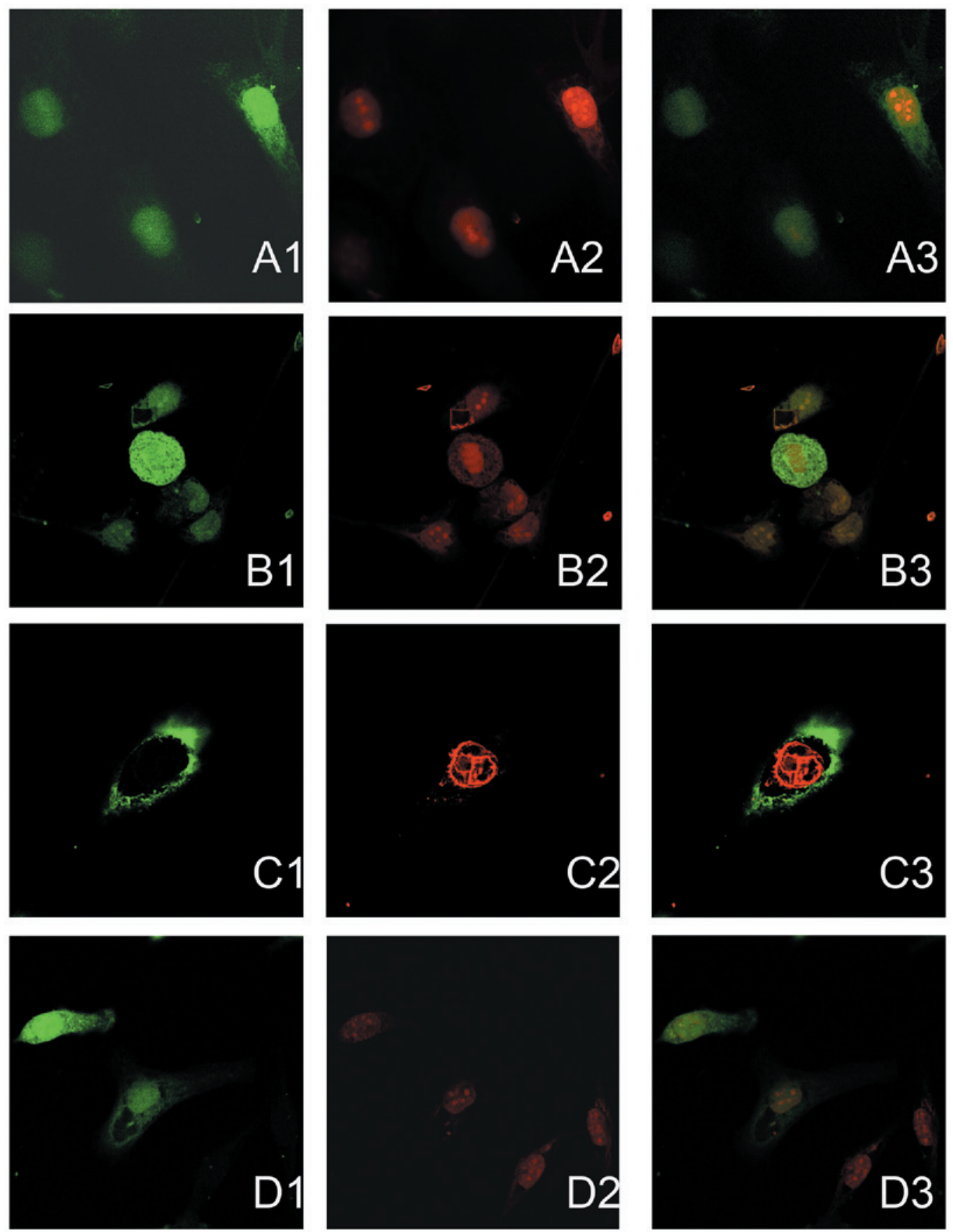

Figure 1. Immunofluorescence staining of PAK4 in four groups (magnification, x400): (A) Untransfected SKOV3 cells; (B) SKOV3 cells transfected by LV3 negative control; (C) SKOV3 cells transfected by LV3-hsa-miR-126 inhibitor; and (D) SKOV3 cells transfected by LV3-hsa-miR-126. (1) Green fluorescent protein-positive cells are indicated by green signals; (2) PAK4-positively stained cells are indicated by red signals; (3) merged images. The mean immunofluorescence intensities of PAK4 in the miR-126 inhibitor group were significantly higher (C2) than that of control group cells (A2), while the expression of PAK4 in the miR-126 upregulated group (D2) was significantly lower compared with that in the control group cells $(\mathrm{P}<0.05)$. PAK, serine/threonine $\mathrm{p} 21$-activated kinase.

the miRNA vectors and PAK4 in the following four groups of SKOV3 cells: Untransfected cells, LV3-NC-transfected cells, LV3-hsa-miR-126-transfected cells and LV3-hsa-miR-126 inhibitor-transfected cells. The expression of PAK4 was indicated by red immunofluorescence staining, and the GFP expressed by the miRNA vectors (green fluorescence) highlighted successfully transfected cells; green fluorescence was detected in all of the nuclei, but only in certain cytoplasmic regions of the SKOV3 cells in the NC, miR-126 inhibitor and miR-126 mimic groups. The mean immunofluorescence intensity of PAK 4 in the miR-126 inhibitor group was significantly higher (Fig. 1, C2) compared with that of the untransfected SKOV3 cells (Fig. 1, A2). Furthermore, the expression level of PAK4 was effectively decreased by the overexpression of miR-126 in the LV3-hsa-miR-126-transfected cells (Fig. 1, D2) compared with that of the untransfected SKOV3 cells (Fig. 1, A2), and particularly compared with that of LV3-hsa-miR-126 inhibitor-transfected cells (Fig. 1, C2). Furthermore, as shown in Fig. $1 \mathrm{C} 3$, the cells transfected with LV3-hsa-miR-126 inhibitor (green) exhibited greater expression of PAK4 (red), whilst cells transfected with 

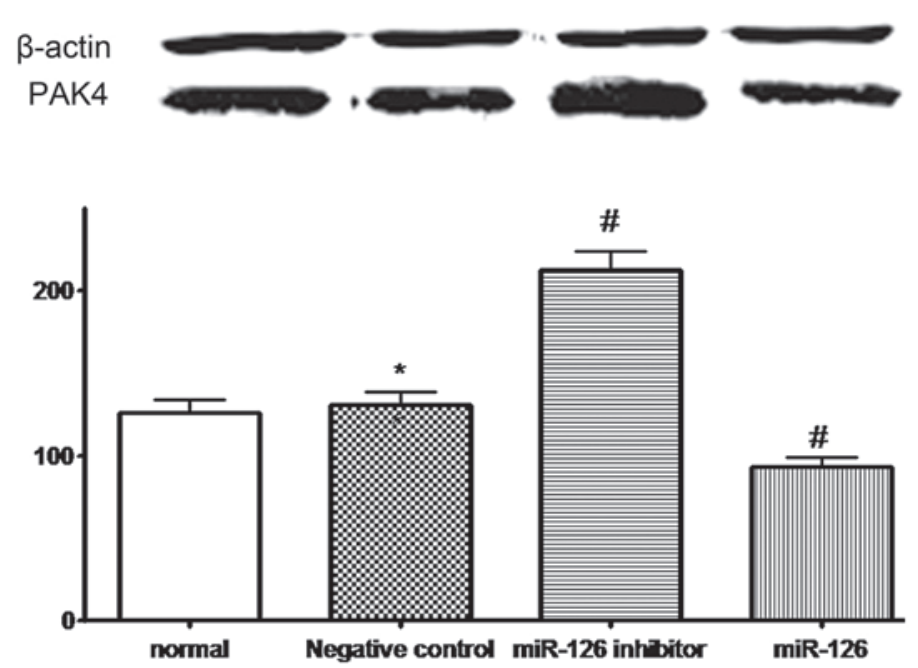

Figure 2. Western blot analysis of PAK4 expression in four groups. Data are presented as the mean \pm standard deviation. Normal, the group of untransfected SKOV3 cells; negative control, the group of SKOV3 cells transfected by LV3 negative control; miR-126 inhibitor, the group of SKOV3 cells transfected with LV3-hsa-miR-126 inhibitor; miR-126, the group of SKOV3 cells transfected by LV3-hsa-miR-126. " $\mathrm{P}>0.05$, vs. normal group, ${ }^{\#} \mathrm{P}<0.05$, vs. normal group. miR/miRNA, microRNA; PAK, serine/threonine p21-activated kinase.

LV3-hsa-miR-126 (green) exhibited reduced expression of PAK4 (red) (Fig. 1 D3).

PAK4 protein expression in the four groups of cells was also evaluated by western blotting. PAK4 was visible as bands of $\sim 64 \mathrm{kDa}$. A densitometric analysis of the PAK $4 / \beta$-actin bands revealed a significant increase in PAK4 expression in the SKOV3 cells transfected with LV3-hsa-miR-126 inhibitor (mean $\pm \mathrm{SD}, 215.1 \pm 10.5$ vs. $128.6 \pm 8.2 \% ; \mathrm{P}=0.001)$ and a decrease in PAK4 expression in the SKOV3 cells transfected with LV3-hsa-miR-126 (mean \pm SD, $91.6 \pm 7.7$ vs. $128.6 \pm 8.2 \%$; $\mathrm{P}=0.002$ ), compared with the untransfected SKOV3 cells (Fig. 2). No significant difference was observed between the expression in the SKOV3 cells in the NC group and those that were untransfected (mean $\pm \mathrm{SD}, 130.9 \pm 9.1$ vs. $128.6 \pm 8.2 \%$; $\mathrm{P}=0.706$; Fig. 2). Therefore, it is proposed that LV3-has-miR-126 inhibitor increases the expression of PAK4, whereas LV3-hsa-miR-126 attenuates this expression.

\section{Discussion}

In the present study, changes in PAK4 expression were demonstrated in ovarian cancer cells with up- or downregulated miR-126 (induced by the transfection of LV-miR-126 or LV-has-miR-126 inhibitor) when compared with normal ovarian cancer cells. The SKOV3 cells transfected with LV-hsa-miR-126 exhibited reduced expression of PAK4, while the cells transfected with LV-hsa-miR-126 inhibitor exhibited increased expression. These findings suggest that miR-126 is a potential tumor suppressor, with the ability to decrease the level of PAK4 in ovarian cancer SKOV3 cells.

The invasive ability of malignant cancer cells depends upon the altered regulation of cell migration by the membrane protrusion formation in response to chemotactic and migratory stimuli (6). Membrane protrusions are formed by polymerization of submembrane actin filaments. The PAK family comprises important signaling proteins that are indicated to be involved in a variety of cellular functions, including cell proliferation, migration and cytoskeletal organization $(7,24)$. The family consists of six members, categorized into two groups: Group A, PAKs 1, 2 and 3; and group B, PAKs 4, 5 and $6(7,25)$. PAK4 has been indicated to be involved in several types of cancer, and strong links have been observed between PAK4 and ovarian cancer (26). Analysis of cell migration and invasion in in vitro and in vivo studies has highlighted the contribution of PAK4 to the progression and metastasis of ovarian cancer; this is consistent with the role of PAK4 in the reorganization of the cytoskeleton and the migration of cells, which is at least in part executed in the cytoplasm (26). PAK4 expression and activation are important in cancer progression, and increased PAK4 expression has been shown to be associated with metastasis, progression to late stages of the disease, reduced patient survival and increased resistance to chemotherapy $(13,14,27)$. The mechanisms by which PAK4 affects ovarian cancer cell progression include the control of cell migration, invasion and proliferation. PAK4 may act via the regulation of c-Src, mitogen-activated protein kinase kinase/extracellular signal-regulated kinases $1 / 2$, matrix metalloproteinase-2, and c-Src/epidermal growth factor receptor. Inhibition of PAK4 may therefore be a potentially valuable therapeutic target $(16,28)$.

miR-126 is a non-coding RNA that is involved in various cellular processes, including proliferation, differentiation, apoptosis and invasion $(17,21,29)$. miRNAs that are upregulated in cancer may function as oncogenes through the negative regulation of tumor suppressor genes, whilst miRNAs that are downregulated may function as tumor suppressor genes and inhibit cancer by regulating oncogenes $(30,31)$. miR-126 acts as a metastatic suppressor in a number of human cancers $(21,23)$. However, the expression and function of miR-126 in ovarian cancer remains unclear. In the present study, the association between miR-126 and PAK4 was investigated in ovarian cancer cells. The results demonstrated that transfection with LV3-miR-126 may efficiently reduce the expression of PAK4 in SKOV3 cells. Furthermore, the LV-miR-126 inhibitor was observed to upregulate the expression of PAK4 in these cells.

In conclusion, as PAK4 is essential for ovarian cancer cell invasion, the present study provides an experimental foundation for the use of miR-126 as a potential tumor suppressor; 
this miRNA may potentially be used to decrease expression levels of PAK4, leading to the inhibition of ovarian cancer cell invasion. However, further studies are required to elucidate the mechanisms involved in the suppression of PAK4 by miR-126.

\section{Acknowledgements}

The authors would like to thank DrLi Yu and DrHongya Wang for theirexcellentassistance. This study was supported by the National Natural Science Foundation of China (grant no. 81371881) and the Science and Technology Department of Zhejiang Province, China (grant no. 2011C23093).

\section{References}

1. Buys SS, Partridge E, Black A, et al; PLCO Project Team: Effect of screening on ovarian cancer mortality: The Prostate, Lung, Colorectal and Ovarian (PLCO) Cancer Screening Randomized Controlled Trial. JAMA 305: 2295-2303, 2011.

2. Matsuda A and Katanoda K: Five-year relative survival rate of ovarian cancer in the USA, Europe and Japan. Jpn J Clin Oncol 44: 196, 2014.

3. Malek JA, Mery E, Mahmoud YA, et al: Copy number variation analysis of matched ovarian primary tumors and peritoneal metastasis. PLoS ONE 6: e28561, 2011.

4. Brandhagen BN, Tieszen CR, Ulmer TM, Tracy MS, Goyeneche AA and Telleria CM: Cytostasis and morphological changes induced by mifepristone in human metastatic cancer cells involve cytoskeletal filamentous actin reorganization and impairment of cell adhesion dynamics. BMC Cancer 13: 35, 2013

5. von Nandelstadh P, Gucciardo E, Lohi J, Li R, Sugiyama N, Carpen $\mathrm{O}$ and Lehti K: Actin-associated protein palladin promotes tumor cell invasion by linking extracellular matrix degradation to cell cytoskeleton. Mol Biol Cell 25: 2556-2570, 2014.

6. Yamaguchi $\mathrm{H}$ and Condeelis J: Regulation of the actin cytoskeleton in cancer cell migration and invasion. Biochim Biophys Acta 1773: 642-652, 2007.

7. Rane CK and Minden A: P21 activated kinases: Structure, regulation, and functions. Small GTPases 5: 5, 2014.

8. Zhu J, Attias O, Aoudjit L, Jiang R, Kawachi H and Takano T: p21-activated kinases regulate actin remodeling in glomerular podocytes. Am J Physiol Renal Physiol 298: F951-F961, 2010.

9. Abo A, Qu J, Cammarano MS, Dan C, Fritsch A, Baud V, Belisle B and Minden A: PAK4, a novel effector for Cdc42Hs, is implicated in the reorganization of the actin cytoskeleton and in the formation of filopodia. EMBO J 17: 6527-6540, 1998.

10. Bompard G, Rabeharivelo G, Cau J, Abrieu A, Delsert C and Morin $\mathrm{N}$ : P21-activated kinase 4 (PAK4) is required for metaphase spindle positioning and anchoring. Oncogene 32: 910-919, 2013.

11. Paliouras GN, Naujokas MA and Park M: PAK4, a novel Gabl binding partner, modulates cell migration and invasion by the Met receptor. Mol Cell Biol 29: 3018-3032, 2009.

12. Dart AE and Wells CM: P21-activated kinase 4 - not just one of the PAK. Eur J Cell Biol 92: 129-138, 2013.

13. Wells CM, Whale AD, Parsons M, Masters JR and Jones GE: PAK4: A pluripotent kinase that regulates prostate cancer cell adhesion. J Cell Sci 123: 1663-1673, 2010.
14. Minden A: The pak4 protein kinase in breast cancer. ISRN Oncol 2012: 694201, 2012.

15. Guo Q, Su N, Zhang J, Li X, Miao Z, Wang G, Cheng M, $\mathrm{Xu} \mathrm{H}$, Cao L and Li F: PAK4 kinase-mediated SCG10 phosphorylation involved in gastric cancer metastasis. Oncogene 33: 3277-3287, 2014.

16. Siu MK, Chan HY, Kong DS, et al: p21-activated kinase 4 regulates ovarian cancer cell proliferation, migration, and invasion and contributes to poor prognosis in patients. Proc Natl Acad Sci USA 107: 18622-18627, 2010.

17. Tokarz $\mathrm{P}$ and Blasiak J: The role of microRNA in metastatic colorectal cancer and its significance in cancer prognosis and treatment. Acta Biochim Pol 59: 467-474, 2012.

18. Zaman MS, Maher DM, Khan S, Jaggi M and Chauhan SC: Current status and implications of microRNAs in ovarian cancer diagnosis and therapy. J Ovarian Res 5: 44, 2012.

19. Luo J, Zhou J, Cheng Q, Zhou C and Ding Z: Role of microRNA-133a in epithelial ovarian cancer pathogenesis and progression. Oncol Lett 7: 1043-1048, 2014.

20. Frampton AE, Krell J, Jacob J, Stebbing J, Castellano L and Jiao LR: Loss of miR-126 is crucial to pancreatic cancer progression. Expert Rev Anticancer Ther 12: 881-884, 2012.

21. Feng R, Chen X, Yu Y, Su L, Yu B, Li J, Cai Q, Yan M, Liu B and Zhu Z: miR-126 functions as a tumour suppressor in human gastric cancer. Cancer Lett 298: 50-63, 2010.

22. Cristóbal I, Aguilera O, García-Foncillas J, Zazo S, Madoz-Gúrpide J and Rojo F: Clinical significance of miR-126 in colorectal cancer. Genes Chromosomes Cancer 53: 881, 2014

23. Sun Y, Bai Y, Zhang F, Wang Y, Guo Y and Guo L: miR-126 inhibits non-small cell lung cancer cells proliferation by targeting EGFL7. Biochem Biophys Res Commun 391: 1483-1489, 2010.

24. Menges CW, Sementino E, Talarchek J, Xu J, Chernoff J, Peterson JR and Testa JR: Group I p21-activated kinases (PAKs) promote tumor cell proliferation and survival through the AKT1 and Raf-MAPK pathways. Mol Cancer Res 10: 1178-1188, 2012.

25. Kumar R, Gururaj AE and Barnes CJ: p21-activated kinases in cancer. Nat Rev Cancer 6: 459-471, 2006.

26. Siu MK, Chan HY, Kong DS, et al: p21-activated kinase 4 regulates ovarian cancer cell proliferation, migration, and invasion and contributes to poor prognosis in patients. Proc Natl Acad Sci USA 107: 18622-18627, 2010.

27. Fu X, Feng J, Zeng D, Ding Y, Yu C and Yang B: PAK4 confers cisplatin resistance in gastric cancer cells via PI3K/Akt- and MEK/Erk-dependent pathways. Biosci Rep 34: 59-67, 2014.

28. Zhang J, Wang J, Guo Q, Wang Y, Zhou Y, Peng H, Cheng M, Zhao D and Li F: LCH-7749944, a novel and potent p21-activated kinase 4 inhibitor, suppresses proliferation and invasion in human gastric cancer cells. Cancer Lett 317: 24-32, 2012.

29. Huang F, Zhu X, Hu XQ, Fang ZF, Tang L, Lu XL and Zhou SH: Mesenchymal stem cells modified with miR-126 release angiogenic factors and activate Notch ligand Delta-like-4, enhancing ischemic angiogenesis and cell survival. Int J Mol Med 31: 484-492, 2013

30. Li L, Huang K, You Y, Fu X, Hu L, Song L and Meng Y: Hypoxia-induced miR-210 in epithelial ovarian cancer enhances cancer cell viability via promoting proliferation and inhibiting apoptosis. Int J Oncol 44: 2111-2120, 2014.

31. Zhang Y, Wang X, Xu B, Wang B, Wang Z, Liang Y, Zhou J, $\mathrm{Hu} \mathrm{J}$ and Jiang B: Epigenetic silencing of miR-126 contributes to tumor invasion and angiogenesis in colorectal cancer. Oncol Rep 30: 1976-1984, 2013. 